\title{
Telomerase inhibition improves tumor response to radiotherapy in a murine orthotopic model of human glioblastoma
}

\author{
Sylvain Ferrandon ${ }^{1,2}$, Céline Malleval ${ }^{3}$, Badia El Hamdani ${ }^{1,4}$, Priscillia Battiston-Montagne ${ }^{1,2}$, Radu Bolbos ${ }^{5}$, \\ Jean-Baptiste Langlois ${ }^{5}$, Patrick Manas ${ }^{6}$, Sergei M Gryaznov ${ }^{7}$, Gersende Alphonse ${ }^{1,4}$, Jérôme Honnorat ${ }^{2,3,8}$, \\ Claire Rodriguez-Lafrasse $e^{1,2,9}$ and Delphine Poncet ${ }^{1,2, *^{*}}$
}

\begin{abstract}
Background: Glioblastoma (GBM) is the most frequent and aggressive type of adult brain tumor. Most GBMs express telomerase; a high level of intra-tumoral telomerase activity (TA) is predictive of poor prognosis. Thus, telomerase inhibitors are promising options to treat GBM. These inhibitors increase the response to radiotherapy (RT), in vitro as well as in vivo. Since typical treatments for GBM include RT, our objective was to evaluate the efficiency of Imetelstat (TA inhibitor) combined with RT.

Findings: We used a murine orthotopic model of human GBM ( $N=8$ to 11 mice per group) and $\mu M R I$ imaging to evaluate the efficacy of Imetelstat (delivered by intra-peritoneal injection) alone and combined with RT. Using a clinically established protocol, we demonstrated that Imetelstat significantly: (i) inhibited the TA in the very center of the tumor, (ii) reduced tumor volume as a proportion of TA inhibition, and (iii) increased the response to RT, in terms of tumor volume regression and survival increase.
\end{abstract}

Conclusions: Imetelstat is currently evaluated in refractory brain tumors in young patients (without RT). Our results support its clinical evaluation combined with RT to treat GBM.

Keywords: Telomerase, Glioma, Irradiation, Orthotopic, GRN163L, Imetelstat, In vivo

\section{Findings}

Reactivation of a telomeric maintenance mechanism is a key and obligatory step for all cancer cells. In fact, telomerase is reactivated in $96 \%$ of cancer tumors. Thus, inhibition of this enzyme is a promising option for treating cancer [1]. GRN163L (Imetelstat) is the most advanced telomerase inhibitor in clinical evaluation (17 completed or ongoing clinical trials) (https://clinicaltrials.gov). In preclinical assays using numerous models of solid and liquid tumors, a reduction in tumor volume and an increase in overall survival (OS) have been reported [2].

In addition, most cancers are treated by radiotherapy (RT), and among the whole genome, $\gamma$-irradiation (IR) preferentially targets telomeres [3] and induces a reduction

\footnotetext{
* Correspondence: delphine.poncet@univ-lyon1.fr

'EMR3738, Cellular and Molecular Radiobiology Laboratory, South Lyon

Charles Mérieux Medicine Faculty, Oullins, France

2Université Claude Bernard Lyon 1, Lyon, France

Full list of author information is available at the end of the article
}

in telomere length [4]. Moreover, shortening telomeres [5-9] increases cell sensitivity to IR, whereas, elongating telomeres induces radio-resistance $[5,10]$. Thus, it makes sense to combine Imetelstat with radiotherapy, as demonstrated in two murine models of breast cancer $[6,11]$.

Glioblastoma (grade IV astrocytoma)(GBM) is the most frequent adult brain tumor, and one of the most aggressive tumors among all human cancers. Despite a standard of care combining surgery, radiotherapy (RT) and Temozolomide chemotherapy, the median patient overall survival (OS) still ranges from 7 to 15 months [12]. New targeted therapies or irradiation techniques are thus urgently needed. However, identification of cancer-specific targets is a particular challenge in GBM as these tumors are highly heterogeneous in terms of their histological, molecular, genetic and epigenetic features [12]. However, most GBMs reactivate telomerase [13-15]. Among these tumors a high intra-tumoral level of telomerase activity 
(TA) $[14,15]$ is predictive of poor prognosis. Two mutations residing in the promoter of TERT (encoding the catalytic subunit of the telomerase) have recently been identified. These mutations, increasing the TA, have been detected in many types of tumors [16], with the highest frequency in GBM (74 \% of patients). Telomerase inhibitors are thus a promising option for treating GBMs.

Drug delivery into the brain is challenging as the Blood Brain Barrier (BBB) stops most of the molecules. Hence, the two first preclinical studies evaluating Imetelstat, in an orthotopic location, delivered the drug directly into the brain $[17,18]$. However, in high grade glioma the BBB is leaky [19], and a more recent publication has provided evidence indicating that Imetelstat passes the BBB [7]. It remains to be demonstrated that the reduction of TA is correlated with a clinical response (such as tumor growth reduction or OS increase). More importantly, RT is used in all GBM treatment plans and Marian et al. have shown, in vitro, on human GBM tumor-initiating cells that 16 weeks of pre-treatment by IMT increases cell death and DNA damage produced by IR. However, the in vivo efficacy of Imetelstat in combination with RT, using a realistic treatment plan, has never been evaluated in GBM.

In this article, we propose, using the intra-peritoneal route in a murine orthotopic model of human GBM, to: (i) demonstrate a reduction in tumor growth in relation with the level of TA inhibition inside the tumor and to (ii) evaluate if Imetelstat increases the efficiency of RT.

Considering the in vivo experiment, we chose a glioblastoma-derived cell line with detectable TA and a telomere length of about $4 \mathrm{~kb}$ (in human TA+ GBM, telomere length ranges from 2 to $11 \mathrm{~kb}$ [13]). This cell line, U87MG, is also the most suitable model for in vivo preclinical assays [20], as it gives rise to tumors in a very reproducible manner. All materials and methods are described in Additional file 1.

First, we wanted to validate the efficiency of the drug alone, before combining it with RT. The U87MG cell line was xenografted in an orthotopic location, and mice were treated by Imetelstat $(\mathrm{N}=8)$ or by the vehicle PBS (Phosphate Buffer Saline) $(\mathrm{N}=8)$, by the intra-peritoneal route, from day 3 (post-xenograft) to euthanasia (Fig. 1a). Twenty-eight days post-graft, we noted a significant reduction in tumor volume (Fig. 1b) with the mice receiving Imetelstat, attesting, for the first time, to the treatment's efficiency when using a peripheral route of injection. However, this efficiency was to be put in relation to the inhibition of the TA. Thus, we measured the TA in the very center of the tumor and observed a significant reduction (Fig. 1c). This confirms that Imetelstat efficiently reaches the center of the tumor. A significant and positive correlation between tumor growth and the residual level of TA was also shown (Fig. 1d). This observation proves: (i) that the anti-tumoral activity of Imetelstat is due to its antitelomerase activity, and (ii) that TA plays an essential role in GBM growth and aggressiveness, reinforcing the interest in targeting telomerase to treat GBM.

We next evaluated the efficiency of the combined treatment with RT, following a plan that would be suitable for human treatment. The mice were treated for

a

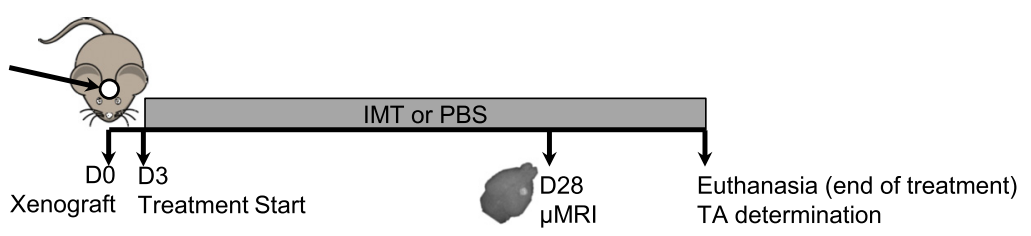

b

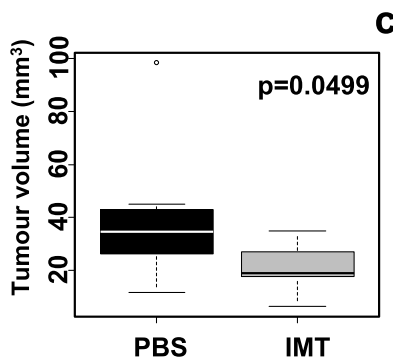

c

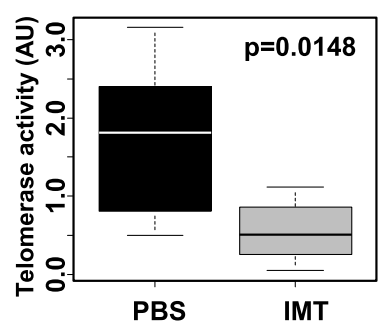

d

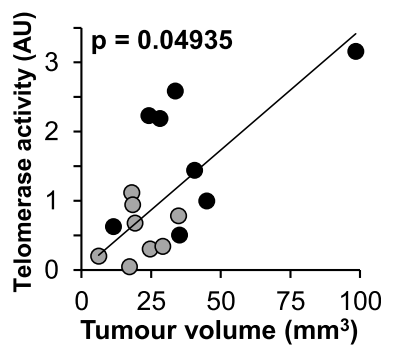

Fig. 1 Intra-peritoneal injection of Imetelstat efficiently inhibits telomerase and reduces tumor growth. a Experimental design: mice were xenografted and intra-peritoneal injections were started three days later, either with Imetelstat (30 mg/Kg three times a week) or by an equivalent volume of PBS. Tumor volume was determined by $\mu \mathrm{MRI}$ at day 28 , and the treatment was maintained until the mice were sacrificed (when tumor growth was predicted to be about $70 \mathrm{~mm}^{3}$ by the $\mu \mathrm{MRI}$ imaging). b Tumor volume at day 28 is significantly reduced by Imetelstat (IMT) treatment versus PBS (Wilcoxon test). $\mathbf{c}$ Intra-peritoneal injection of Imetelstat is able to significantly reduce the TA inside the tumor (Wilcoxon test). $\mathbf{d}$ TA and tumor volume are correlated (Spearman test), the grey and black circles correspond respectively to the mice treated by IMT or by PBS 
one month with Imetelstat and RT was delivered concomitantly, two weeks post induction (as validated by our in vitro results, data not shown). The RT protocol was a focalized IR of the brain, five times a week by $2 \mathrm{~Gy}$ fractions (as used for humans) for one week (Fig. 2a). On day 26, a significant reduction in tumor volume was observed by $\mu \mathrm{MRI}$, in comparison with the PBS control group (PBS), regardless of the treatment : Imetelstat (IMT, $\mathrm{p}=$ 0.0084), PBS plus RT (PBS/RT, $\mathrm{p}=0.0053)$, or Imetelstat plus RT (IMT/RT, p=0.0004) (Figs. 2c, d). As observed in our in vitro experiments (data not shown), we noted that Imetelstat significantly increased the efficiency of RT, in term of tumor volume reduction $(\mathrm{p}=0.0414)$ (Figs. 2c, $\mathrm{d}$ ). As expected, the OS was increased in all 3 treatments (PBS/RT, IMT or IMT/RT) (Fig. 2b left). If considering the IMT/RT versus the PBS/RT groups we also established a significant $(\mathrm{p}=0.036)$ increase in OS (Fig. $2 b$ right). The median OS was respectively 30, 39, 39 and 41 days for the

\section{a}

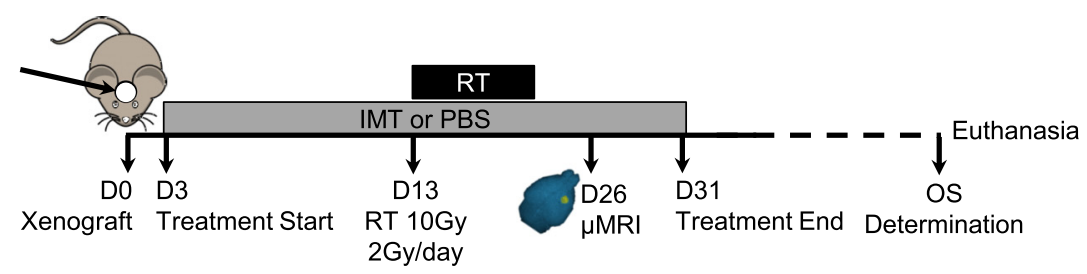

b
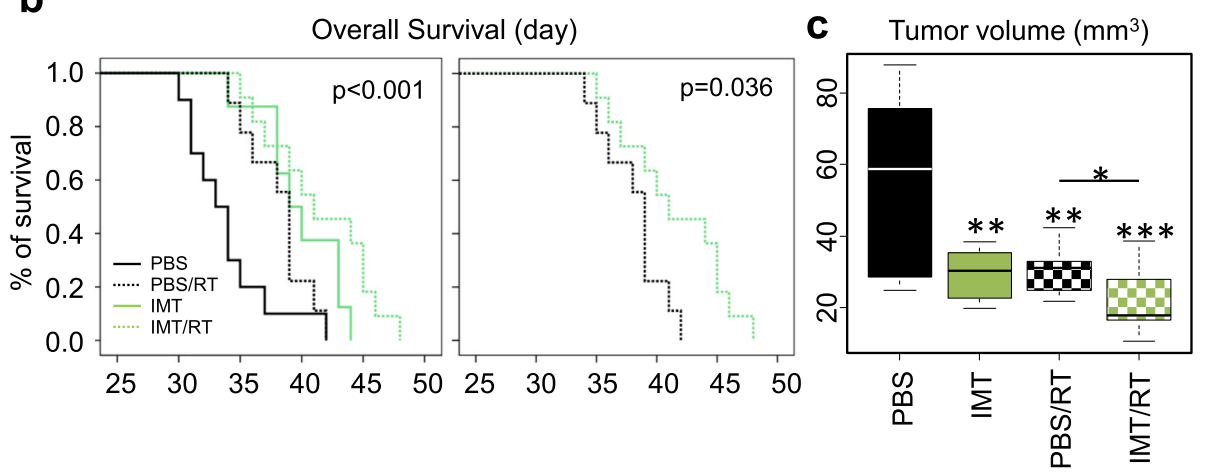

d

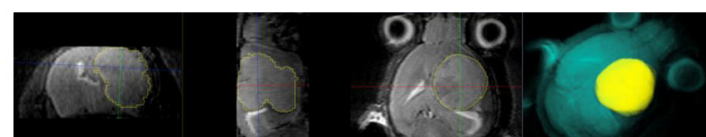

PBS

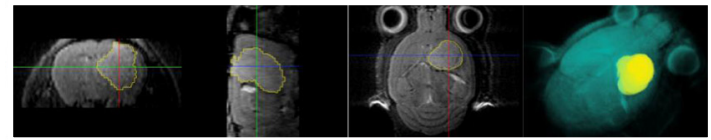

IMT

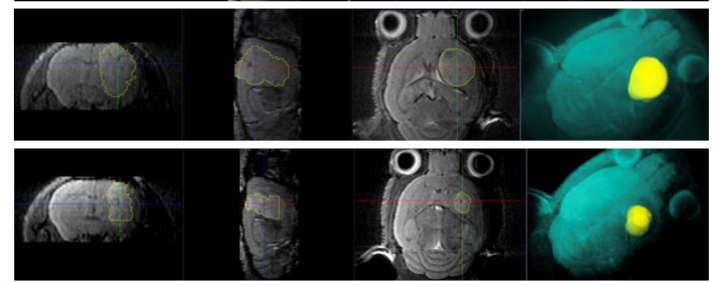

PBS/RT

IMT/RT

Fig. 2 Imetelstat significantly increases radiotherapy efficiency in vivo. a Experimental design: mice were xenografted (D0) and intra-peritoneal injections were started three days later (D3) either with Imetelstat (IMT, $30 \mathrm{mg} / \mathrm{Kg}$ three times a week) or by an equivalent volume of PBS, for four weeks. Two weeks after the treatment was began (D13) mice were or were not concomitantly treated by radiotherapy (RT, 2Gy/day, five days), and imaged by $\mu M R I$ at day 26 (D26). Injections were stopped at the end of the fourth week and mice were monitored until they developed debilitating disease (date used for the OS). b Kaplan Meier representation of OS as a function of days post-xenograft, for mice treated by PBS (black solid line), Imetelstat (green solid line) or a combination of RT with PBS (dashed black line) or with Imetelstat (dashed green line). LogRank calculated p-values are shown for all treatments (on the left) and for IMT/RT versus PBS/RT (on the right). c Tumor volume determined by $\mu \mathrm{MRI}$ at day 26 is shown for each treatment group. p-values were determined comparing each condition to the PBS condition, and between the PBS/RT versus the IMT/RT $\left(p<0.05\right.$, ${ }^{* *} p<0.01$, ${ }^{* * *} p<0.001$, Wilcoxon test). $\mathbf{d}$ Display of semi-automatic GBM segmentation on T2-weighted MR images: axial plane (left), sagittal plane (middle), coronal plane (right). A 3D reconstruction (right) showing the localization and the size of GBM (yellow) within the mouse brain (turquoise) 
PBS, IMT, RT and IMT/RT conditions. Thanks to a standard curve of tumoral growth (Additional file 2: Figure S1), we have translated these results into tumoral volume variation: the combined treatment reduced the growth by $34 \%$ in comparison to IMT or RT alone. Furthermore, $45 \%$ of mice (5 over 11 mice) in the IMT/RT group have the same or an increased OS than mice in the RT or IMT group. To conclude, the combination of RT with Imetelstat significantly reduced tumor volumes and increased the OS of the mice.

Considering the results obtained with Imetelstat alone, we showed a significant reduction in TA, in the very center of the tumor, and established that the residual TA was positively correlated with the tumor's growth. This highlights the major role of TA in these tumors' aggressiveness in agreement with previous publications setting a correlation between patient outcome and tumoral-TA level $[14,15]$. In the future, dose optimization could be proposed to patients, in proportion to the initial TA level inside their tumors or their circulating tumoral cells [21]. While, RT is an obligatory step for GBM treatment, no pre-clinical data was available concerning the combination of RT and Imetelstat for GBM treatment. We have demonstrated a significant improvement of the efficiency of RT, by combining it with Imetelstat for GBM treatment, in terms of OS increase and tumor volume reduction. It is noteworthy that we have used RT for only one week to avoid the radical effect of intensive RT that would have precluded any evaluation of its combined effect with Imetelstat (as in Serrano et al. publication [8]). Thus, we expect that the effect of the combination will be even more drastic with several weeks of concomitant RT (as commonly delivered in human GBM treatment). Moreover, we did not observe any side effect, confirming the results of previous preclinical assays [2].

Last, a major point of our model is that it avoids most of the inconsistencies of previous publications, such as the long-term cell pre-treatment before engraftment [11, 22], or the concomitant treatment together with engraftment [18], both of which preclude tumor engraftment and development [23]. This anti-engraftment or anti-adhesive [24] effect could be related to the off-target effect of Imetelstat on cytoskeleton destructuration [25]. Moreover, we propose a realistic protocol for clinical use: (i) a peripheral route of injection and not a direct intra-cerebral [18], intra-nasal [17] or intra-tumoral [11] delivery, (ii) a classical one-month cycle of chemotherapy and (iii) a combined fractionated RT treatment (2Gy/fraction delivered locally). Overall, our data supports the usefulness of future clinical studies using Imetelstat combined with RT for the treatment of GBM. The recent opening of a clinical trial evaluating Imetelstat (alone) in refractory brain tumors in young patients comforts this conclusion (NCT01836549).

\section{Additional files}

\section{Additional file 1: Materials and methods. \\ Additional file 2: Determination of tumor growth after U87} orthotopic xenoraft.

\section{Abbreviations}

GBM: Glioblastoma; TA: Telomerase activity; RT: Radiotherapy; OS: Overall survival; IR: $\gamma$-irradiation; IMT: Imetelstat.

\section{Competing interests}

The authors declare that they have no competing interests.

\section{Authors' contributions}

$\mathrm{SF}, \mathrm{BEH}$ and PBM carried out the in vivo experiments and the preliminary in vitro setting. CM carried out all the xenografts and helped set the in vivo experimental design. JBL and RB performed the $\mu \mathrm{MRI}$ and calculated the tumoral volume. PM was in charge of animal caring. CRL proofread the manuscript. SG provided the drug and helped design the experimental plan. GA and $\mathrm{JH}$ participated in the design of the study and proofread the manuscript. DP conceived of and coordinated the study, participated to the in vivo experiments, performed the statistical analyses and drafted the manuscript. All authors read and approved the final manuscript.

\section{Acknowledgments}

We thank the «Association pour la Recherche contre le Cancer » (ARC), the « Association des Neuro-Oncologues d'Expression Française » (ANOCEF) and « La Ligue Nationale contre le Cancer » (comité de la Saône) for their financial support. We thank Geron Corp. for supporting our research (grant and gift of Imetelstat), Pierre Verrelle for his gift of cell lines, Guillaume Belz for his help in "R" manipulation, Uzma Hasan, Noor Alkurd and Stéphanie Simonet for English proofreading and the AniRA technical platforms of US8/UMS3444. The $\mu M R I$ imaging was performed on CERMEP - imagerie du vivant, Bron, F-69677, France, imaging facilities. This work was achieved within the scientific framework of Labex-PRIMES (ANR-11LABX-0063) and the laboratory was supported by the PRRH (Programme Régional de Recherches en Hadronthérapie). The manuscript has been copy edited by Anchor English corp.

\section{Author details}

${ }^{1}$ EMR3738, Cellular and Molecular Radiobiology Laboratory, South Lyon Charles Mérieux Medicine Faculty, Oullins, France. ${ }^{2}$ Université Claude Bernard Lyon 1, Lyon, France. ${ }^{3}$ Team « neuro-oncology and neuro-inflammation », Lyon Neuroscience Research Center, INSERM U1028/CNRS UMR 5292, Laennec Medicine Faculty, Lyon, France. ${ }^{4}$ Hospices Civils de Lyon, South Lyon Hospital, Pierre Bénite, France. ${ }^{5}$ CERMEP-imagerie du vivant, Groupement Hospitalier Est, Bron, France. ${ }^{6}$ UMS 3444 gerland CNRS P.B.E.S - Ecole Normale Supérieure Lyon, Lyon, France. ${ }^{7}$ Geron Corporation, 149 Commonweath Drive, Menlo Park, CA 94025, USA. ${ }^{8}$ Department of neuro-oncology, Hospices civils de Lyon, Bron, France. ${ }^{9}$ Biochemistry Department, Transfer and Molecular Oncology Unit, South Lyon Hospital, Hospices Civils de Lyon, Pierre Bénite, France.

Received: 29 January 2015 Accepted: 1 May 2015

Published online: 17 July 2015

\section{References}

1. Harley CB. Telomerase and cancer therapeutics. Nat Rev Cancer. 2008;8:167-79.

2. Ouellette MM, Wright WE, Shay JW. Targeting telomerase-expressing cancer cells. J Cell Mol Med. 2011;15:1433-42.

3. Oikawa S, Kawanishi S. Site-specific DNA damage at GGG sequence by oxidative stress may accelerate telomere shortening. FEBS Lett. 1999;453:365-8.

4. von Zglinicki T, Pilger R, Sitte N. Accumulation of single-strand breaks is the major cause of telomere shortening in human fibroblasts. Free Radic Biol Med. 2000;28:64-74.

5. Ferrandon S, Saultier P, Carras J, Battiston-Montagne P, Alphonse G, Beuve M, Malleval C, Honnorat J, Slatter T, Hung N, et al. Telomere Profiling: Toward Glioblastoma Personalized Medicine. Mol Neurobiol. 2013;47(1):64-76.

6. Gomez-Millan J, Goldblatt EM, Gryaznov SM, Mendonca MS, Herbert BS. Specific telomere dysfunction induced by GRN163L increases radiation sensitivity in breast cancer cells. Int J Radiat Oncol Biol Phys. 2007;67:897-905. 
7. Marian CO, Cho SK, McEllin BM, Maher EA, Hatanpaa KJ, Madden CJ, et al. The telomerase antagonist, imetelstat, efficiently targets glioblastoma tumor-initiating cells leading to decreased proliferation and tumor growth. Clin Cancer Res. 2010;16:154-63.

8. Serrano D, Bleau AM, Fernandez-Garcia I, Fernandez-Marcelo T, Iniesta P, Ortiz-de-Solorzano C, et al. Inhibition of telomerase activity preferentially targets aldehyde dehydrogenase-positive cancer stem-like cells in lung cancer. Mol Cancer. 2011;10:96.

9. Goytisolo FA, Samper E, Martin-Caballero J, Finnon P, Herrera E, Flores JM, et al. Short telomeres result in organismal hypersensitivity to ionizing radiation in mammals. J Exp Med. 2000;192:1625-36.

10. Akiyama M, Yamada O, Kanda N, Akita S, Kawano T, Ohno T, et al. Telomerase overexpression in $\mathrm{K} 562$ leukemia cells protects against apoptosis by serum deprivation and double-stranded DNA break inducing agents, but not against DNA synthesis inhibitors. Cancer Lett. 2002;178:187-97.

11. Weng D, Cunin MC, Song B, Price BD, Eller MS, Gilchrest BA, et al. Radiosensitization of mammary carcinoma cells by telomere homolog oligonucleotide pretreatment. Breast Cancer Res. 2010;12:R71.

12. Preusser M, de Ribaupierre S, Wohrer A, Erridge SC, Hegi M, Weller M, et al. Current concepts and management of glioblastoma. Ann Neurol. 2011;70:9-21.

13. Hakin-Smith V, Jellinek DA, Levy D, Carroll T, Teo M, Timperley WR, et al. Alternative lengthening of telomeres and survival in patients with glioblastoma multiforme. Lancet. 2003;361:836-8.

14. Lotsch D, Ghanim B, Laaber M, Wurm G, Weis S, Lenz S, et al. Prognostic significance of telomerase-associated parameters in glioblastoma: effect of patient age. Neuro Oncol. 2013;15:423-32.

15. Tchirkov A, Rolhion C, Kemeny JL, Irthum B, Puget S, Khalil T, et al. Clinical implications of quantitative real-time RT-PCR analysis of hTERT gene expression in human gliomas. Br J Cancer. 2003;88:516-20.

16. Killela PJ, Reitman ZJ, Jiao Y, Bettegowda C, Agrawal N, Diaz Jr LA, et al. TERT promoter mutations occur frequently in gliomas and a subset of tumors derived from cells with low rates of self-renewal. Proc Natl Acad Sci U S A. 2013;110:6021-6.

17. Hashizume R, Ozawa T, Gryaznov SM, Bollen AW, Lamborn KR, Frey 2nd WH, et al. New therapeutic approach for brain tumors: Intranasal delivery of telomerase inhibitor GRN163. Neuro Oncol. 2008;10:112-20.

18. Ozawa T, Gryaznov SM, Hu L, Pongracz K, Santos RA, Bollen AW, et al Antitumor effects of specific telomerase inhibitor GRN163 in human glioblastoma xenografts. Neuro Oncol. 2004;6:218-26.

19. Roberts HC, Roberts TP, Brasch RC, Dillon WP. Quantitative measurement of microvascular permeability in human brain tumors achieved using dynamic contrast-enhanced MR imaging: correlation with histologic grade. AJNR Am J Neuroradiol. 2000;21:891-9.

20. de Vries NA, Beijnen JH, van Tellingen O. High-grade glioma mouse models and their applicability for preclinical testing. Cancer Treat Rev. 2009;35:714-23.

21. Goldkorn A, Ely B, Tangen CM, Tai YC, Xu T, Li H, et al. Circulating tumor cell telomerase activity as a prognostic marker for overall survival in SWOG 0421: a phase III metastatic castration resistant prostate cancer trial. Int J Cancer. 2015;136:1856-62.

22. Joseph I, Tressler R, Bassett E, Harley C, Buseman CM, Pattamatta P, et al. The telomerase inhibitor imetelstat depletes cancer stem cells in breast and pancreatic cancer cell lines. Cancer Res. 2010;70:9494-504.

23. Castelo-Branco P, Zhang C, Lipman T, Fujitani M, Hansford L, Clarke I, et al. Neural tumor-initiating cells have distinct telomere maintenance and can be safely targeted for telomerase inhibition. Clin Cancer Res. 2011;17:111-21.

24. Jackson SR, Zhu CH, Paulson V, Watkins L, Dikmen ZG, Gryaznov SM, et al. Antiadhesive effects of GRN163L-an oligonucleotide N3'- > P5' thiophosphoramidate targeting telomerase. Cancer Res. 2007;67:1121-9.

25. Mender I, Senturk S, Ozgunes N, Akcali KC, Kletsas D, Gryaznov S, et al. Imetelstat (a telomerase antagonist) exerts offtarget effects on the cytoskeleton. Int J Oncol. 2013;42:1709-15.

\section{Submit your next manuscript to BioMed Central and take full advantage of:}

- Convenient online submission

- Thorough peer review

- No space constraints or color figure charges

- Immediate publication on acceptance

- Inclusion in PubMed, CAS, Scopus and Google Scholar

- Research which is freely available for redistribution

Submit your manuscript at www.biomedcentral.com/submit 